\title{
Jeunes et prises de risque sportives. Vers une approche sociologique contextualisée
}

\section{Bastien Soulé et Jean Corneloup}

\section{(2) OpenEdition \\ 1 Journals}

\section{Édition électronique}

URL : http://journals.openedition.org/corpsetculture/471

DOI : 10.4000/corpsetculture.471

ISSN : $1777-5337$

\section{Éditeur}

Association Corps et Culture

\section{Édition imprimée}

Date de publication : 1 juin 1998

ISSN : 1268-5631

\section{Référence électronique}

Bastien Soulé et Jean Corneloup, « Jeunes et prises de risque sportives. Vers une approche sociologique contextualisée », Corps et culture [En ligne], Numéro 3 | 1998, mis en ligne le 24 septembre 2007, consulté le 08 septembre 2020. URL : http://journals.openedition.org/ corpsetculture/471 ; DOI : https://doi.org/10.4000/corpsetculture.471

Ce document a été généré automatiquement le 8 septembre 2020.

(c) tous droits réservés 


\title{
Jeunes et prises de risque sportives. Vers une approche sociologique contextualisée
}

\author{
Bastien Soulé et Jean Corneloup
}

Introduction

1 Comment peut-on aborder la thématique du risque corporel tout en traitant du lien social ? La réponse à cette question n'irait pas de soi si cette réflexion n'avait déjà été envisagée, voilà exactement un siècle, par Durkheim (1897). Son approche princeps sur les mobiles du suicide montre en effet que ceux-ci sont directement corrélés au niveau d'intégration sociale des individus. Ce paradigme fonctionnaliste a par la suite servi de référence à un domaine élargi par rapport aux préoccupations premières du père de la sociologie française : celui du traitement des déviances, de la délinquance, et plus globalement des " conduites à risque ». Sans remettre en cause sa pertinence, il parait nécessaire, sous l'impulsion des récentes généralisations et diversifications des comportements risqués au sein de la jeunesse française, de développer un nouveau type d'approche. L'exemple de l'engagement dans une pratique sportive dite "à risque » le montre : il est non seulement librement consenti, mais souvent aussi le fait d'individus bien insérés socialement (Mitchell, 1983; Duret, 1996; Le Breton, 1996). Ce qui met à mal les théories explicatives centrées sur une quelconque forme de défaut de socialisation. L'abord de la question du risque sportif rend en conséquence nécessaire l'élargissement du champ de vision, afin de dépasser les approches partielles, inclinées vers un déterminisme peu adapté à la perspective globale envisagée ici.

2 Ce questionnement a pour origine un double constat: l'avènement d'un style sportif nouveau ${ }^{1}$, dont les jeunes sont les principaux acteurs, au sein duquel le risque occupe une place grandissante ${ }^{2}$ et la récente recrudescence, chez les jeunes, de comportements à risque plus « classiques " (alcoolisation excessive, toxicomanie, tentatives de suicide, conduite automobile imprudente, violences, etc. $\left.{ }^{3}\right)$. On voit là se dessiner les prémices d'un champ, au sens de Bourdieu (1984), de la prise de risque juvénile. L'objectif est de mieux le cerner, en s'efforçant d'aller au delà de son aspect factuel, pour mettre à jour 
les variables explicatives des comportements en question et les relations entre style de vie et pratique à risque.

Cadre de la recherche

3 Tout d'abord, il apparaît nécessaire de préciser quelques termes récurrents et de dresser un état des lieux succinct des comportements évoqués.

Le risque

4 Bien qu'il soit considéré comme un " nid de concepts ", il est tout de même possible de mettre en avant quelques traits génériques de ce terme. Le risque est avant tout affaire de potentialité. C'est l'incertitude qui caractérise donc le mieux cette notion. L'événement, s'il est malheureux, n'est que la réalisation de la probabilité d'apparition du danger, et constitue s'il est heureux la moisson effective des gains espérés lors de l'adoption de la conduite incertaine. Le danger, c'est la possibilité qu'un accident survienne, lui même caractérisé par la nature de ses dommages et leur gravité.

Il convient également de rappeler l'ambivalence sociale du terme, à la fois potentiellement négatif et bénéfique. Ce qui engendrera dans le premier cas une sécurisation et une aseptisation de l'existence parfois poussées jusqu'à l'absurde, alors que l'on parlera dans le second cas de "prise de risque », ou de choix (opportuniste) relatif à l'engagement dans une situation plus ou moins incertaine. Le risque n'a dans cette optique plus rien de contingent; il est désiré et provoqué. Un «plus », un profit d'ordre quelconque justifie ces comportements et rend le danger acceptable. Plusieurs formes d'attitude face au risque sont donc envisageables. Il peut alors être question, pour ce qui est des prises de risque corporelles, de « comportement autodestructeur de compromis » (Assailly, 1990, p. 101) quand le dommage est prévu mais non désiré, par opposition à l'accident (le danger n'est ni prévu ni désiré) ou encore au suicide, à l'anorexie (le danger est prévu et désiré).

6 En ce qui concerne les pratiques sportives dites à risque (sports de glisse, V.T.T., parapente, "skate-board", escalade, etc.), celles-ci ne sont pas toutes physiquement plus dangereuses que les sports que l'on qualifie aujourd'hui de classiques (tels que le football, le rugby, la gymnastique, etc.) $)^{4}$. Ce sont les représentations associées à ces activités qui jouent par rapport à ce pôle un rôle d'amplificateur; côtoiement de la mort, figures héroïques et exploits mythiques sont relayés par une médiatisation croissante. Le risque est un concept abstrait, socialement construit par les individus. Sa définition s'apparente souvent à un enjeu. Ce travail consistera donc, entre autre, en une tentative de compréhension des rapports au risque socialement différenciés. Nous nous démarquons, en adoptant cette position relativiste, d'une part des approches objectives et probabilistes issues de l'accidentologie sportive (Collard, 1997), et d'autre part des définitions extrêmes du risque corporel, centrées sur son pendant mortel. Ce dernier n'étant pas exclusif de toute autre forme de risque corporel (entorses, fractures, contusions ou autres traumatismes). De même, le risque corporel n'est pas la seule modalité envisageable de risque sportif: les dommages peuvent être de nature tactique (mauvais choix de l'entraîneur), technique (tentative infructueuse d'un geste hors du commun) ou encore interactionnelle (exposition de sa motricité au regard de tiers). Le constat sous-jacent à cette parenthèse est celui de la diversité de la prise de risque sportive, comparable à celle du risque en général (Jackson et al., 1972). Il n'y a donc a priori aucune raison pour circonscrire cette analyse au versant corporel des prises de risque sportives, même si celles-ci représentent la clé de voûte et le principal centre d'intérêt du travail entrepris. 
La nouvelle configuration de la jeunesse

7 Le statut d'adolescent, en dehors de toute considération conjoncturelle, est en lui même porteur d'ambiguïté sociale. « Mi-enfant mi-adulte », mais « ni enfant ni adulte " (Bourdieu, 1984, p. 146), les jeunes sont confrontés à une situation transitoire caractérisée par le changement, la déstabilisation. Il s'agit là d'un invariant anthropologique, que certaines sociétés tentent de conjurer et d'éradiquer par des rites de passage ou des rites initiatiques.

8 Sans entrer dans les détails de la situation actuelle de la jeunesse, on peut de plus évoquer le moratoire social qui s'est installé en son sein, dû à une " entrée dans la vie " (Galland, 1991, p.121) de plus en plus tardive et difficile. La jeunesse n'est plus simplement issue de la crise adolescente, mais bien de la crise « tout court 》 (Marange, 1994, p. 30). On parle d'ailleurs à son propos de " génération miroir " (Padis, 1996, p. 5) tant elle reflète à travers ses déboires la situation transitoire de notre fin de siècle. Ce temps de latence est souvent vécu difficilement sur le plan psychologique, générant une sorte de «mal être » multiforme, une forme d'inquiétude voire de désarroi dans la façon d'envisager l'avenir. Constat également valable pour les jeunes présentant le moins de difficultés sociales ${ }^{5}$.

On est tout naturellement porté à s'interroger sur les conséquences purement sociales de ces nouvelles données. Le modèle de l'héritage social est quelque peu bouleversé. Un éclatement s'est produit, qui donne à voir une multitude de situations. L'objet de notre étude rend pourtant nécessaire la traduction en termes scientifiques de ces dernières. Il est possible de distinguer, dans ce but, trois états ${ }^{6}$. En premier lieu, l'intégration sociale, qui se caractérise par l'existence de liens avec les quatre pôles intégrateurs que représentent le travail, l'école, les communautés et la famille. L'exclusion désignant, elle, le processus conduisant à la rupture d'au moins un des liens évoqués (abandon scolaire, inactivité professionnelle de longue durée, rupture des relations avec la famille, etc.). Enfin, une situation intermédiaire, que nous appellerons «instabilité sociale ", semble se généraliser en parallèle avec l'évolution du contexte décrite plus haut. Elle est le fruit d'une fragilisation d'un ou plusieurs liens avec les quatre pôles cités, générant des déséquilibres, une forme d'incertitude quant à l'avenir. Ce type de classification permet de cerner non seulement les jeunes bien insérés, les jeunes exclus (les "galériens", auxquels nous nous intéresserons peu dans ce travail en raison de leur faible degré d'investissement dans une pratique sportive) mais également les personnes en situation d'attente. On évite ainsi tout réductionnisme dichotomique mettant face à face le monde des "in-group ", parfaitement intégrés à la société, et celui des exclus, des "out group» vivant à sa marge, confrontés à la misère, la délinquance ou la drogue. Ce modèle complexifié de la jeunesse permettra de rendre compte avec plus de finesse des divergences comportementales face au problème étudié.

Le champ des prises de risque corporelles de la jeunesse

10 La vie dans la société française actuelle est sans aucun doute plus confortable pour la jeunesse qu'elle ne le fut au début du siècle, ou a fortiori à des périodes plus éloignées comme le $\mathrm{XIX}^{\mathrm{e}}$ siècle ou le moyen-âge. On ne part plus à la guerre, la violence est peu ou prou domestiquée, la précocité du travail et la pénibilité de la tâche tendent à diminuer, la médecine progresse régulièrement... Pourtant, il semblerait que le rapport au risque de la jeunesse ait changé, qu'il soit moins subi, plus délibéré. On évoque souvent à ce propos les « conduites à risque $»^{7}$, comme la toxicomanie ou l'alcoolisation 
excessive. Ce sont elles qui viennent aujourd'hui en premier lieu à l'esprit quand on évoque les risques menaçant la jeunesse. C'est bien là le signe d'un changement de la perception des risques auxquels les jeunes sont exposés. D'autres prises de risque corporelles sont à l'inverse valorisées socialement. C'est le cas de la plupart des pratiques sportives dites "à risque ", dont le visage est jugé plus présentable. Voici, sous forme de tableau, quelques éléments constitutifs du champ évoqué. Ils sont séparés en trois parties et présentés au sein de chacune d'elles, le moins arbitrairement possible, par ordre décroissant de « dangerosité » corporelle.

\section{Conduites motrices intentionnellement risquées}

\begin{tabular}{|c|c|}
\hline $\begin{array}{l}\text { Conduite } \\
\text { automobile } \\
\text { risquée }\end{array}$ & $\begin{array}{l}\text { Source de mortalité } \mathrm{N}^{\circ} 1 \text { chez les } 15-14 \text { ans }(3 / 4 \text { des hommes et } 3 / 5 \text { des } \\
\text { femmes décédant chaque année). Surexposition en tous points au danger }{ }^{8} \text {. }\end{array}$ \\
\hline Suicide & $\begin{array}{l}\text { 2ème cause de mortalité chez les } 15-24 \text { ans }{ }^{9} \text {. Nombre de tentatives en hausse, } \\
\text { surtout chez les jeunes en difficulté. }\end{array}$ \\
\hline Violences & $\begin{array}{l}\text { «Bastons » entre jeunes de quartiers sensibles identifiées comme le principal } \\
\text { vecteur des conduites à risque juvéniles }{ }^{10} \text {. }\end{array}$ \\
\hline $\begin{array}{l}\text { Comportements } \\
\text { sportifs à risque }\end{array}$ & $\begin{array}{l}\text { Un jeune homme sur deux déclare avoir eu un accident sportif au cours de } \\
\text { l'année précédente }{ }^{11} \text {, et le nombre de traumatismes aux membres déclarés } \\
\text { lors des tests de sélection militaire a doublé de } 1987 \text { à } 1993^{12} \text {. Même si l'on n'a } \\
\text { pas d'information précise quant à la répartition exacte de ces accidents par } \\
\text { type de sport }{ }^{13} \text {, il est indéniable que certaines modalités récemment apparues } \\
\text { sont plus « accidentogènes » que les sports dits classiques }{ }^{14} \text {. }\end{array}$ \\
\hline
\end{tabular}

\section{Consommations à caractère risqué ou illicite}

\begin{tabular}{|l|l|}
\hline Alcoolisme & $\begin{array}{l}\text { Développement «toxicomaniaque }{ }^{15} \text { de l'usage de l'alcool. Augmentation de la } \\
\text { fréquence de l'alcoolisation, des quantités ingérées, précocité croissante de } \\
\text { l'alcoolisation }{ }^{16} \text {. }\end{array}$ \\
\hline \hline Tabagisme & $\begin{array}{l}\text { A } 16 \text { ans, un jeune sur deux fume. A } 18 \text { ans on atteint } 60 \% \text { de fumeurs, plus haut } \\
\text { niveau enregistré depuis } 1990 \text { et record de l'union européenne }{ }^{17} \text {. }\end{array}$ \\
\hline \hline Toxicomanie & $\begin{array}{l}\text { Consommation de cannabis identifiée comme un trait marquant caractérisant la } \\
\text { jeunesse actuelle }{ }^{18} \text {. Pratique «de jeune ", avant tout masculine, elle tend à se } \\
\text { généralise }{ }^{19} \text { (les autres substances, telles que l'héroïne, la cocaïne ou les } \\
\text { amphétamines, sont rarement adoptées par les moins de } 30 \text { ans). }\end{array}$ \\
\hline
\end{tabular}




\begin{tabular}{|l||l|}
\hline $\begin{array}{l}\text { Comportements } \\
\text { sexuels à risque }\end{array}$ & $\begin{array}{l}\text { Seuls } 13 \% \text { des jeunes en difficulté utilisent un préservatif avec des } \\
\text { partenaires inhabituels. De plus, ou en conséquence de cette négligence, } \\
10 \% \text { des filles dans cette situation ont déjà pratiqué une I.V.G. }{ }^{20}\end{array}$ \\
\hline \hline Divers & $\begin{array}{l}\text { "décibelisme » (avec un walkman, en boite de nuit ou en « rave party »), " } \\
\text { piercing ", négligences vis-à-vis de sa santé, mauvaise alimentation... }\end{array}$ \\
\hline
\end{tabular}

11 Cette diversité des passages à l'acte ou consommations potentiellement risqués pour l'organisme augure bel et bien de l'existence d'un champ de la prise de risque juvénile. Quelques-uns des comportements évoqués sont de plus l'objet d'une interdiction légale, faisant de leur adoption une exposition à un double danger. On observe des chiffres supérieurs a ceux constatés pour le reste de la population, et en hausse par rapport aux années précédentes. Une incontestable stratification sociale des risques physiques se dessine. La bonne santé physique des jeunes, constatée par ailleurs ${ }^{21}$, est en fait pour partie d'entre eux sciemment altérée par ces comportements.

Apport théorique et problématique

Une fois les bases de notre approche posées, nous avons cherché à étudier les liens, les relations entre la jeunesse, ses divers visages et le champ des prises de risque juvéniles mis à jour. La première phase de ce travail consiste en une analyse bibliographique, qui met en évidence une opposition entre deux cadres théoriques dominants portant sur la perception et la fonction du risque. Plusieurs champs disciplinaires, outre la sociologie, sont mis à contribution. Ainsi, l'anthropologie ou la psychologie apportent des éclairages complémentaires, que le fait d'ignorer serait réducteur.

Le risque pathologique : l'exposition de son corps à un danger, dans ce premier cas, est envisagée comme une sorte de déviance, un comportement anormal adopté en réponse à un environnement social particulier. Les comportements à risque sont alors perçus comme des réponses, des palliatifs à une situation anomique, a une sorte d'instabilité sociale, voire à une trop grande intégration. Dans cette optique, on n'est pas «normal » quand on s'engage dans des comportements risqués. De moins en moins régulés par une quelconque forme institutionnelle de ritualisation (Le Breton, 1991), ceux-ci s'imposent au regard extérieur comme un stigmate ou la preuve d'un « mal être ».

\begin{tabular}{|l||l||l|l|}
\hline Types de risque & $\begin{array}{l}\text { Appuis théoriques, } \\
\text { conditions de validité }\end{array}$ & $\begin{array}{l}\text { Auteurs de } \\
\text { référence }\end{array}$ & Apports théoriques \\
\hline \hline \begin{tabular}{l|l|l|} 
LE RISQUE \\
ANOMIQUE
\end{tabular} & $\begin{array}{l}\text { Théorie fonctionnaliste } \\
\text { Affaiblissement des liens } \\
\text { sociaux }\end{array}$ & $\begin{array}{l}\text { ESTERLE } \\
\text { HEDIBEL } \\
(1995)\end{array}$ & $\begin{array}{l}\text { Culture corporelle du risque chez } \\
\text { les jeunes de banlieue. } \\
\text { Délinquance adrénaline, } \\
\text { présentéisme, ère du «tout tout } \\
\text { de suite ». }\end{array}$ \\
\hline
\end{tabular}




\begin{tabular}{|c|c|c|c|}
\hline & $\begin{array}{l}\text { Contexte familial et } \\
\text { scolaire défavorable }\end{array}$ & $\begin{array}{l}\text { JESSOR } \\
(1989)\end{array}$ & $\begin{array}{l}\text { "Théorie des comportements } \\
\text { problèmes ». Conduite à risque } \\
\text { commer syndromer de } \\
\text { comportements de santé à risque. }\end{array}$ \\
\hline & $\begin{array}{l}\text { Intégration rociale } \\
\text { incomplète, de } r \text { type } \\
\text { communautaire, limitée à } \\
\text { la bande }\end{array}$ & $\begin{array}{l}\text { HIRSCHI } \\
(1969)\end{array}$ & $\begin{array}{l}\text { "Théorie du contrôle»: risques } \\
\text { socialement acceptés (sportifs par } \\
\text { ex.) pour les jeunes intégrés, } \\
\text { risques déviants pour ceux dont } \\
\text { l'intégration se limite à la bande. }\end{array}$ \\
\hline $\begin{array}{l}\text { LE RISQUE } \\
\text { STRATIFIE }\end{array}$ & Théorie de l'habitus & $\begin{array}{l}\text { BOLTANSKI } \\
(1971)\end{array}$ & $\begin{array}{l}\text { Exposition fréquente de sa santé, } \\
\text { absence de prévention, tolérance } \\
\text { à la douleur élevée dues à un } \\
\text { habitus corporel populaire. }\end{array}$ \\
\hline $\begin{array}{l}\text { LE RISQUE } \\
\text { ORDALIQUE }\end{array}$ & $\begin{array}{l}\text { Crise anthropologique } \\
\text { contemporaine. Perte du } \\
\text { sens, des valeurs, des } \\
\text { repères surplombants }\end{array}$ & $\begin{array}{l}\text { LE BRETON } \\
(1991)\end{array}$ & $\begin{array}{l}\text { Tests de son existence, recherche } \\
\text { du sens de la vie. Conduites } \\
\text { ordaliques. }\end{array}$ \\
\hline \multirow[t]{2}{*}{$\begin{array}{l}\text { LE RISQUE } \\
\text { STRATEGIQUE }\end{array}$} & Théorie de Bourdieu & $\begin{array}{l}\text { POCIELLO } \\
(1981)\end{array}$ & $\begin{array}{l}\text { Réaction face à la chute ou au } \\
\text { déclassement sociaux. Rêve de } \\
\text { «vol social ». }\end{array}$ \\
\hline & $\begin{array}{llr}\text { Frustration } & \text { de } & \text { la } \\
\text { «bourgeoisie } & & \text { de } \\
\text { compétence » } & \text { face } & \text { aux } \\
\text { agents dotés } & \text { d'un } & \text { fort } \\
\text { capital économique. } & \end{array}$ & $\begin{array}{l}\text { BOURDEAU } \\
(1995)\end{array}$ & $\begin{array}{l}\text { Compensation par la } \\
\text { confrontation au signifiant noble } \\
\text { qu'est la mort, dont la valeur est, } \\
\text { elle, inestimable. }\end{array}$ \\
\hline \multirow[t]{3}{*}{$\begin{array}{l}\text { LE } \\
\text { DEROUTINSANT }\end{array}$} & Théorie de la rupture & $\begin{array}{l}\text { ELIAS \& } \\
\text { DUNNING } \\
(1994)\end{array}$ & $\begin{array}{l}\text { Mimétisme, «jeu de rôle ". On } \\
\text { cherche à changer de peau, à se } \\
\text { glisser dans celle d'un héros. }\end{array}$ \\
\hline & $\begin{array}{l}\text { Intégration excessive, } \\
\text { situation sociale trop } \\
\text { stable, liberté entravée } \\
\text { (« déterminismes } \\
\text { inversés ») }\end{array}$ & $\begin{array}{l}\text { MITCHELL } \\
(1983) \\
\text { LYNG } \\
(1990)\end{array}$ & $\begin{array}{l}\text { Respiration, besoin d'aventure. } \\
\text { Pratiques déroutinisantes, comme } \\
\text { l'alpinisme ou le vol libre (donner } \\
\text { le meilleur de soi, enfin). }\end{array}$ \\
\hline & 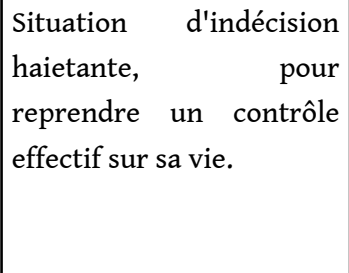 & $\begin{array}{l}\text { KLAUSNER } \\
(1968) \\
\text { SACHS } \\
(1990)\end{array}$ & $\begin{array}{l}\text { Jeux de hasard, logique de } \\
\text { l'indécision. Désir de maîtrise, de } \\
\text { contrôle d'un problème tangible, } \\
\text { facilementridentifiable, } \\
\text { contrairement aux problèmes } \\
\text { quotidiens plus complexes. }\end{array}$ \\
\hline
\end{tabular}




\begin{tabular}{|l|l|l|l|l|}
\hline $\begin{array}{l}\text { LE RISQUE } \\
\text { CATHARSIS }\end{array}$ & $\begin{array}{l}\text { Etats tensionnels, } \\
\text { agressivité accumulés. }\end{array}$ & $\begin{array}{l}\text { ASSAILLY } \\
(1992)\end{array}$ & $\begin{array}{l}\text { Extériorisation des stress, } \\
\text { compensation des frustrations par } \\
\text { l'action. }\end{array}$ \\
\hline \hline $\begin{array}{l}\text { L'EXCELLENCE } \\
\text { PAR LE RISQUE }\end{array}$ & $\begin{array}{l}\text { Pathologie du gagneur, } \\
\text { de l'excellence à tout prix } \\
\text { et en tout. }\end{array}$ & $\begin{array}{l}\text { EHRENBERG } \\
(1991)\end{array}$ & $\begin{array}{l}\text { «ulte de la performance » } \\
\text { transposé aux activités sportives } \\
\text { et de loisir. }\end{array}$ \\
\hline \hline & $\begin{array}{l}\text { AUBERT \& } \\
\text { DE GALLEJAC } \\
(1984)\end{array}$ & $\begin{array}{l}\text { Logique entrepreneuriale de } \\
\text { l'excellence transposée au } \\
\text { domaine extra-professionnel. }\end{array}$ \\
\hline
\end{tabular}

Le risque dynamique : à l'opposé de ces déterminismes, au sens large, on peut envisager la prise de risque de façon positive. L'attitude change alors du tout au tout, dans le sens où celle ci va devenir un moyen destiné à atteindre un bénéfice quelconque, sans forcément, comme dans le premier cadre, être dicté par un élément du contexte social. Le risque devient alors l'élément d'un mode de vie placé sous le signe du jeu, du ressenti, La pratique d'un sport à risque, au sens premier du terme, rentre a priori dans ce cadre. Comme Le Breton (1996) a pu le montrer, elle est souvent le fait d'individus jouissant d'une intégration sociale des plus satisfaisantes, à l'inverse des prises de risque pathologiques mentionnées ci-dessus. Le risque peut donc être envisagé, en dehors de tout déterminisme ou compensation sociaux, comme un filtre herméneutique de la réalité, permettant une sorte de «mieux être». A condition d'accepter de miser au moins sur le plan symbolique, son intégrité corporelle. C'est cette vision commune de la prise de risque comme possibilité d'atteindre une certaine harmonie qui relie les points de vue théoriques ci-dessous.

Le risque dynamique

\begin{tabular}{|c|c|c|c|}
\hline $\begin{array}{l}\text { Types de } \\
\text { risque }\end{array}$ & $\begin{array}{l}\text { Appuis théoriques, } \\
\text { conditions de validité }\end{array}$ & $\begin{array}{l}\text { Auteurs de } \\
\text { référence }\end{array}$ & Apports théoriques \\
\hline \multirow[t]{4}{*}{$\begin{array}{l}\text { LE RISQUE } \\
\text { RITUEL }\end{array}$} & $\begin{array}{l}\text { Phase psychologique du } \\
\text { développement } \\
\text { pubertaire Théorisation } \\
\text { de la socialisation } \\
\text { juvénile }\end{array}$ & $\begin{array}{l}\text { ASSAILLY } \\
(1992)\end{array}$ & $\begin{array}{l}\text { Recherche de "containing", de } \\
\text { limites, d'autonomie à l'adolescence. } \\
\text { Expérimentation de son nouveau } \\
\text { corps. }\end{array}$ \\
\hline & & $\begin{array}{l}\text { POCIELLO } \\
(1992)\end{array}$ & $\begin{array}{l}\text { Rite de passage, quête initiatique } \\
\text { favorisés par l'aventure, le risque. } \\
\text { Turbulence juvénile. }\end{array}$ \\
\hline & $\begin{array}{l}\text { Psychologie de la } \\
\text { construction identitaire }\end{array}$ & $\begin{array}{l}\text { TAP } \\
(1996)\end{array}$ & $\begin{array}{l}\text { Marquer sa différence, «se } \\
\text { construire » en se distinguant. }\end{array}$ \\
\hline & $\begin{array}{l}\text { Se signaler en } \\
\text { s'opposant, en affinant } \\
\text { ses spécificités }\end{array}$ & $\begin{array}{l}\text { LORET } \\
(1995)\end{array}$ & $\begin{array}{l}\text { Logique de la transgression, tests de la } \\
\text { société, opposer la "glisse" et le } \\
\text { sport... }\end{array}$ \\
\hline
\end{tabular}




\begin{tabular}{|c|c|c|c|}
\hline $\begin{array}{l}\text { LE RISQUE } \\
\text { TRAGIQUE }\end{array}$ & $\begin{array}{l}\text { Sociologie du tragique } \\
\text { Anthropologie de la } \\
\text { mort }\end{array}$ & $\begin{array}{l}\text { BAUDRY } \\
(1991) \\
\text { GRIFFET } \\
(1991)\end{array}$ & $\begin{array}{l}\text { Nécessité d'apprivoiser, de jouer avec } \\
\text { la mort, cette altérité qui fonde le } \\
\text { rapport au monde. Délimitation plus } \\
\text { précise du réel et de soi. Signe d'un } \\
\text { « vouloir vivre» }\end{array}$ \\
\hline $\begin{array}{lr}\text { LE } & \text { RISQUE } \\
\text { "MODE } & \text { DE } \\
\text { VIE" } & \end{array}$ & Théorie de la culture & $\begin{array}{l}\text { CORNELOUP } \\
(1990)\end{array}$ & $\begin{array}{l}\text { Expression d'un mode de vie, } \\
\text { prédispositions à l'aventure. Partage } \\
\text { d'émotions communes, socialité } \\
\text { autour du risque. }\end{array}$ \\
\hline $\begin{array}{l}\text { LE RISQUE } \\
\text { EMOTION }\end{array}$ & $\begin{array}{l}\text { Théorie esthétique, } \\
\text { recherche de sensations }\end{array}$ & $\begin{array}{l}\text { ZUCKERMAN } \\
(1979) \\
\text { LORET } \\
(1995) \\
\text { ASSAILLY } \\
(1992)\end{array}$ & $\begin{array}{l}\text { Les jeunes sont des «sensation } \\
\text { seekers ». Stimulation, plaisir } \\
\text { recherchés. «Culture fun», prise de } \\
\text { risque prétexte à la sensation, au } \\
\text { «frisson». }\end{array}$ \\
\hline $\begin{array}{lr}\text { LE RISQUE } \\
\text { DES VALEURS }\end{array}$ & Théorie des conflits & $\begin{array}{l}\text { PAGES \& } \\
\text { CORNELOUP } \\
(1990)\end{array}$ & $\begin{array}{l}\text { Cohérence entre les comportements } \\
\text { adoptés et les valeurs du changement, } \\
\text { du mouvement, de la transgression. } \\
\text { Idéologie libertaire. }\end{array}$ \\
\hline \multirow[t]{3}{*}{$\begin{array}{l}\text { LE RISQUE } \\
\text { IDENTITAIRE }\end{array}$} & $\begin{array}{l}\text { Microsociologie, } \\
\text { interactionnisme } \\
\text { symbolique }\end{array}$ & $\begin{array}{l}\text { ASSAILLY } \\
(1992) \\
\text { GOFFMAN } \\
(1974)\end{array}$ & $\begin{array}{l}\text { Risque comme signifiant identitaire, } \\
\text { communicationnel. } \\
\text { stratégique dans les relations de face- } \\
\text { à-face. }\end{array}$ \\
\hline & $\begin{array}{l}\text { Théorie bourdieusienne } \\
\text { du champ, stratégies } \\
\text { spécifiques }\end{array}$ & $\begin{array}{l}\text { POCIELLO } \\
(1981)\end{array}$ & $\begin{array}{l}\text { Pratique d'un sport à risque comme } \\
\text { élément distinctif au sein du système } \\
\text { des sports. }\end{array}$ \\
\hline & $\begin{array}{l}\text { Désir de reconnaissance } \\
\text { des plus démunis } \\
\text { symboliquement }\end{array}$ & $\begin{array}{l}\text { EHRENBERG } \\
(1991) \\
\text { THERME } \\
(1995) \\
\text { REY } \\
(1993)\end{array}$ & $\begin{array}{l}\text { Désir d'appartenance au monde des } \\
\text { vainqueurs pour les «exclus de la } \\
\text { performance " (scolaire notamment), } \\
\text { grâce à des prises de risque destinées } \\
\text { à «être quelqu'un». Ne plus être } \\
\text { personne... }\end{array}$ \\
\hline $\begin{array}{l}\text { LE RISQUE } \\
\text { POST- } \\
\text { MODERNE }\end{array}$ & Théorie post-moderne & $\begin{array}{l}\text { MAFFESOLI } \\
(1997,1991)\end{array}$ & $\begin{array}{l}\text { Accomplissement de soi grâce au } \\
\text { côtoiement du risque, de l'aventure et } \\
\text { de la mort. Errance, vagabondages } \\
\text { permettant le développement d'une } \\
\text { personnalité vitale. }\end{array}$ \\
\hline
\end{tabular}




\begin{tabular}{|l|l|l|l|l|}
\hline \begin{tabular}{ll|l|l|} 
LE RISQUE \\
COGNITIF
\end{tabular} & $\begin{array}{l}\text { Individualisme } \\
\text { méthodologique } \\
\text { Psychologie cognitive }\end{array}$ & $\begin{array}{l}\text { DELIGNIERES } \\
(1993)\end{array}$ & $\begin{array}{l}\text { Notion de risque préférentiel (rapport } \\
\text { maximal entre les bénéfices et les } \\
\text { coûts liés au comportement adopté), } \\
\text { Maitrise de soi, de ses possibilités. Jeu } \\
\text { rationnel avec le support de la } \\
\text { pratique. Stratégie informationnelle. }\end{array}$ \\
\hline
\end{tabular}

15 C'est dans la diversité de ces options théoriques que réside l'intérêt de l'approche. Cette juxtaposition d'explications parfois opposées est révélatrice de la complexité du phénomène étudié. Il s'agira, à partir de cette prise de conscience, de rendre compte des différentes constructions sociales, des logiques parfois contradictoires conduisant vers l'adoption de tel ou tel comportement constitutif du champ de la prise de risque juvénile.

16 La position sociale, le niveau d'intégration sociale des jeunes exercent certainement une influence sur leur positionnement au sein du dit champ. Cette correspondance est une sorte de truisme; elle nous servira avant tout de point de départ. Elle sous-entend l'existence de déterminismes et de stratégies autour de notre objet, dont la nature reste néanmoins à déterminer.

17 Toutefois, il convient de dépasser ce stade pour s'intéresser à un deuxième niveau, plus finement explicatif. Comme le laissent présager les éléments abordés dans la partie propédeutique de ce travail (qu'ils soient empiriques ou théoriques), il serait abusif de considérer les comportements et attitudes relatifs au risque comme étant figés à l'intérieur de ce cadre s'apparentant au "structuralisme génétique " de Bourdieu (1987). Des nuances sont à apporter. La réalité n'est pas constituée, c'est là notre première hypothèse, de situations binaires, entièrement déterminées socialement. Il y a tout intérêt à faire preuve de relativisme. Cet affinement n'est en rien un déni de toute influence macrosociale: l'analyse se situe en fait dans une perspective sociologique constructiviste (Corcuff, 1995), proche de la «sociologie de l'expérience» de Dubet (1994). L'éclatement du social et des logiques d'action individuelles contraint à moduler en quelque sorte, ce qui pourrait rendre un caractère trop statique à l'abord du phénomène.

18 Ce positionnement implique, enfin, une troisième remarque : seule une approche de type microsociologique permettra de rendre compte du caractère plus ou moins opérant, selon le public, de chaque variable explicative issue du cadre théorique. De plus, cette option méthodologique devrait permettre de tester une seconde hypothèse : l'importance des variables contextuelles sur ces prises de risque, notamment à travers l'interaction sociale qui semble en être une dimension souvent oubliée. Lopes (1983) est l'un des rares chercheurs à s'être intéressé au facteur situationnel des conduites à risque, lequel repose selon lui sur les réponses des individus à des besoins immédiats et des opportunités. Certains chercheurs ont d'ailleurs d'ores et déjà choisi de s'intéresser à cette microsociologie sportive. Parmi eux, Ohl (1993) réinvestit la «théâtralisation de soi» de Goffman (1973) pour expliquer les comportements consommatoires en matière sportive. Dans une optique prophylactique, Fournier et al. (1996) ont souligné la valeur d'échange des conduites à risque. Le preneur de risques s'inscrivant dans une «économie transactionnelle» de la prise de risque, à l'intérieur de laquelle le risque est 
utilisé pour sa portée interpellante. Il est possible d'élargir cette capacité communicative des prises de risque à celles qui sont calculées, dynamiques.

Résultats de l'étude

19 Pour rendre compte des hypothèses de travail, des entretiens semi-directifs ont été réalisés avec deux groupes caractéristiques, composés chacun d'une douzaine d'individus : le premier comprend des jeunes « risqueurs » sportifs, a priori bien insérés (Bali, 1972) («skateurs», surfeurs, "snowboarders», vététistes ...), et le second des jeunes « socialement instables » pratiquant une activité sportive (basket ou foot de rue principalement). On note une nette sur-représentation masculine, alors que l'âge des interviewés est variable (de 15 à 24 ans). Au nom de l'« expérience», les pratiques respectives de ces deux groupes construisent aussi le social, et permettront de prendre la mesure des divergences supposées dans le rapport au risque spécifique à chaque population. La technique de recueil de données a été choisie en raison de son adéquation à notre démarche compréhensive, censée rendre compte des diverses constructions sociales dont le risque pouvait être l'objet. Le nombre d'entretiens n'a pas été choisi aléatoirement mais déterminé par l'atteinte de la phase de saturation des modèles. Celle-ci se caractérise par la faiblesse puis l'absence d'éléments nouveaux apportés par les derniers entretiens réalisés. On peut alors légitimement penser qu'une certaine forme de représentativité, en rien statistique mais explicative, a été atteinte. C'est d'ailleurs un des arguments techniques avancés par Mucchielli (1991) pour attester de la validité scientifique de l'entretien comme méthode de recueil de données. Chaque entretien a ensuite été retranscrit par écrit, puis une analyse thématique a permis de ventiler les propos des interviewés selon les thèmes recensés lors de la construction du guide d'entretien: pratique sportive personnelle, type et niveau de risque perçus de celle-ci, définition du risque, des comportements à risque, conduites personnelles considérées comme risquées, justifications éventuelles de ces dernières et enfin influence de l'environnement sur ces prises de risque. Il s'agit donc d'un traitement horizontal des données recueillies, ou procédure «par boites » (Bardin, 1980).

20 Pour des nécessités de concision, cette partie ne sera abordée qu'à travers la comparaison des résultats obtenus auprès des deux groupes. Elle permettra de confronter le cadre théorique mis sur pied au réel. Cette présentation comporte cinq rubriques. La première concerne les prises de risque déclarées (niveau purement factuel). Puis on a cherché à savoir, à travers la définition du risque et la façon d'envisager l'investissement personnel dans un comportement risqué, comment chaque groupe se positionnait par rapport aux deux pôles de notre cadre théorique. Les deux dernières parties du tableau servant à la meilleure compréhension des justifications et du contexte de ces prises de risque. Pour chaque thème retenu, le tableau suivant permet une visualisation rapide des points communs (en gras) et des points divergents (en italique) révélés par l'analyse de contenu des entretiens réalisés. Les données qui suivent ne sont que des résultats appelés à évoluer, et en rien des conclusions définitives. 
Synthèse des points communs et des différences pertinentes apparues entre le groupe des jeunes " risqueurs » sportifs et celui des jeunes sportifs socialement instables.

\begin{tabular}{|c|c|c|}
\hline & « RISQUEURS » SPORTIFS INTEGRES & $\begin{array}{l}\text { SPORTIFS } \\
\text { SOCIALEMENT INSTABLES }\end{array}$ \\
\hline Prises de risque & $\begin{array}{l}\text { Légères prises de risque sportives } \\
\text { corporelles (en skate, roller, VTT } \\
\text { etc.) Consommations illicites } \\
\text { fréquentes (« cuites », «shit », etc.) } \\
\text { « Rien à prouver au volant» }\end{array}$ & $\begin{array}{l}\text { Prises de risque sportives d'ordre } \\
\text { tactico-technique en foot, basket } \\
\text { de rue Consommations illicites } \\
\text { rares Vitesse, acrobaties en } \\
\text { scooter ou vélo }\end{array}$ \\
\hline Définition du risque & $\begin{array}{l}\text { Restreinte au risque violent, létal } \\
\text { (accident, maladie mortels) Risque } \\
\text { plutôt associé à l'importance du } \\
\text { danger couru qu'à sa probabilité } \\
\text { d'apparition }\end{array}$ & $\begin{array}{l}\text { Lecture élargie, plus réaliste, avec } \\
\text { prise en compte des effets } \\
\text { secondaires du tabac, de l'alcool, } \\
\text { etc. }\end{array}$ \\
\hline $\begin{array}{l}\text { Perception, conception } \\
\text { de l'engagement } \\
\text { personnel dans une } \\
\text { conduite à risque }\end{array}$ & $\begin{array}{l}\text { Exagération du risque déclaré } \\
\text { dans la pratique sportive } \\
\text { personnelle } \\
\text { Volonté de contrôle, de maîtrise du } \\
\text { risque pris. Refus du hasard, de } \\
\text { l'ordalie comme juge unique }\end{array}$ & $\begin{array}{l}\text { Logique emphatique dans le } \\
\text { discours relatif aux prises de } \\
\text { risque personnelles } \\
\text { Prises de risque plus aléatoires, } \\
\text { analogiques }\end{array}$ \\
\hline $\begin{array}{l}\text { Signification, fonction } \\
\text { de la prise de risque }\end{array}$ & $\begin{array}{l}\text { Recherche de profits symboliques } \\
\text { par le biais de la prise de risque } \\
\text { Recherche de sensations fortes } \\
\text { (Jeunes «sensation seekers"). Le } \\
\text { but : «s'éclater" } \\
\text { "Consumation» corporelle, à des } \\
\text { fins de plaisir. Morale hédoniste } \\
\text { Positionnement stratégique, } \\
\text { identitaire (risque sportif utilisé } \\
\text { comme « signifiant-maître ") } \\
\text { Idée de progression par le risque } \\
\text { (aller au-delà de soi) } \\
\text { Test de ses capacités }\end{array}$ & $\begin{array}{l}\text { Recherche de profits } \\
\text { symboliques et matériels, } \\
\text { professionnels par le risque } \\
\text { Idem, principalement grâce à la } \\
\text { vitesse } \\
\text { Rentabilisation corporelle } \\
\text { (recherche de performance, de } \\
\text { gains.. .) } \\
\text { Risque sportif «identitaire ». } \\
\text { valorisant (Rey, 1993) } \\
\text { Exercer un contrôle sur la } \\
\text { situation, rarement effectif au } \\
\text { quotidien ("réussir») } \\
\text { Rôle de "rassurance» }\end{array}$ \\
\hline
\end{tabular}




\begin{tabular}{|c|c|c|}
\hline $\begin{array}{l}\text { Influence des variables } \\
\text { situationnelles }\end{array}$ & $\begin{array}{l}\text { Groupe activateur de la prise de } \\
\text { risque, surenchère par le défi. } \\
\text { Rôle activateur } \\
\text { Consommation conviviale de } \\
\text { cannabis ou d'alcool, lors de soirées } \\
\text { entre pairs } \\
\text { Recherche fréquente de mise en } \\
\text { spectacle, de théâtralisation } \\
\text { Effet recherché au sein du groupe et } \\
\text { sur l'assistance, même inconnue et } \\
\text { mouvante Logique de «haut lieu " } \\
\text { du "skate park» situé en centre } \\
\text { Ville }\end{array}$ & $\begin{array}{l}\text { Idem } \\
\text { Idem } \\
\text { Communication avant tout } \\
\text { tournée vers le pôle local (le } \\
\text { quartier et ses résidents). } \\
\text { Immédiateté des retombées } \\
\text { espérées } \\
\text { Pas de terrain fédérateur pour les } \\
\text { basketteurs de rue }\end{array}$ \\
\hline
\end{tabular}

\section{Commentaires et interprétation}

21 L'objet de cette troisième partie sera d'aller au-delà de la simple présentation des résultats, pour atteindre un niveau plus proprement explicatif. Le caractère éclaté des variables explicatives des prises de risque rend peu évidente la séparation des différentes constructions constatées. Il y a bien cependant un jeu de subtiles différences qui apparaît entre les deux groupes, sur lequel il convient d'insister. Dans un premier temps, le cheminement thématique de la synthèse des résultats sera suivi. Par la suite, seront abordés les freins à la pratique d'un sport à risque corporel au sein de la population socialement instable.

Types de prise de risque

On cherchera à expliquer les différences comportementales perceptibles dans la confrontation au risque. Quelques précisions semblent nécessaires avant d'aller plus loin dans la compréhension du phénomène étudié.

Il parait important, tout d'abord, de relativiser le niveau de danger objectif auquel se frottent les sujets sportifs "à risque » interviewés. L'on n'a en effet pas eu affaire à des jeunes se frottant à des dangers extrêmes. Soulignons ensuite la nature du risque sportif auquel se confrontent les jeunes socialement instables, lorsqu'ils s'adonnent à la pratique du « street-ball » ou du foot de rue : il est plutôt d'ordre technique ou tactique, destiné à prouver, à soi-même et aux autres « interactants ", ce dont on est capable. La définition volontairement large du risque adoptée pour ce travail permet de prendre en compte ces prises de risque spécifiques. Le risque majeur est alors celui de " perdre la face » (Goffman, 1973). L'usage fait de la pratique sportive implique en conséquence des comportements audacieux, garantissant une certaine visibilité, un impact sur l'entourage. On note, non sans surprise, une faible propension au sein de ce second groupe à mettre son intégrité corporelle en jeu.

Quel que soit le groupe, on est proche de l'absence de danger physique véritable (dans le sens de la mise en jeu de son intégrité corporelle).

Définition du risque

L'origine du décalage perceptible entre les deux groupes est facilement explicable d'un point de vue sociologique. En effet, les jeunes bien intégrés bénéficient d'un environnement social protecteur, sur lequel ils s'appuient et peuvent compter Leur vision de l'avenir, sans être rose, est porteuse d'espoir, de projets. Les sources de 
danger sont alors perçues comme lointaines, rares, accidentelles. (« Le risque violent, ce qui t'tombe sur le coin d'la gueule... »).

A l'inverse, la « précarité » à laquelle sont confrontés les jeunes socialement instables développe chez ces derniers une forme de "paranoïa » qui génère un affinement, une plus grande prise en compte des facteurs de risque. D'où les mentions faites aux risques secondaires inhérents à certaines consommations, ou encore aux dangers domestiques, quotidiens. (« Partout y'a du risque... ») (« La vie elle est bourrée de risque, hein... »).

Perception de l'engagement personnel dans une conduite à risque

Si l'on a pu parler d'une relative homologie entre les deux groupes sur le thème du discours attenant aux prises de risque juvéniles, certaines subtilités peuvent être décelées, qui laissent filtrer quelques différences. S'il est incontestable que les prises de risque, notamment corporelles, permettent grâce à leur forte capacité signifiante une valorisation personnelle, cette dernière n'est pas exactement recherchée de la même manière selon le public. Plutôt directe, emphatique dans le discours des jeunes socialement instables ( J'descends l'viaduc à fond, 90/100 km/h... J'me dis j'suis fou ! J'pourrais m'tuer!») et davantage construite chez les « sujets à risque » sportifs. Ceuxci préfèrent simplement souligner le caractère risqué de leur pratique, de manière anodine, ce qui, au vu de leur définition du risque, est tout à fait édifiant. Ce subterfuge permet de se positionner sur le pôle du risque sportif sans pour autant verser dans les envolées dithyrambiques associées à autre public.

28 A propos de la volonté de contrôle du risque pris chez les "risqueurs » sportifs, on remarque que quand cette emprise sur les faits devient improbable, le comportement n'est généralement pas adopté. On retrouve là cette approche cognitiviste du risque théorisée par Delignières (1993). Le risque perçu semble tout à fait jouer, au sein de cette population, le rôle pondérateur qui est le sien. Ce côté réfléchi, mesuré des prises de risque s'applique d'ailleurs à des domaines extra-sportifs (comme la conduite automobile). Au contraire, les jeunes «socialement instables" investis dans des conduites à risque, plus rares, le sont de manière beaucoup plus analogique, sans précautions particulières, en scooter par exemple (vitesse élevée, absence de casque...

Fonctions de la prise de risque

Le vertige, le « jeu aux limites » (Griffet, 1991, p. 362) procure au sein des deux groupes contactés des sensations qui justifient en partie les comportements corporellement risqués. Le vecteur utilisé pour satisfaire ces "sensation seekers" (Zuckerman, 1979) sera simplement différent selon les groupes (cf. «type de prises de risque »). Cette recherche est particulièrement privilégiée chez les «risqueurs» sportifs, qui font fréquemment état de leur désir de profiter «à fond » de leur jeunesse et de leur bonne santé, de "s'éclater» avant d'atteindre un âge qui infirmera leurs possibilités physiques d'épanouissement. Ce qui passe par diverses modalités activatrices de ce plaisir. («C'est génial de prendre des risques... On s'éclate ») (« ... J'me sens tout drôle, j'ai envie de recommencer »). C'est dans ce sens qu'il est question de "consumation » de l'existence, de souhait de "profiter» de sa jeunesse, au risque d'exposer son organisme à diverses violences passives... Tout ceci dans une forme de douce quiétude, d'insouciance quant à l'avenir.

Bien qu'ils soient eux aussi présents sur le pôle de la recherche de sensations, les jeunes socialement instables ont plutôt le souci, de manière générale, de rentabiliser leur capital corporel. Certains vont même jusqu'à condamner vertement le tabagisme, la consommation d'alcool ou de drogue, qui infirmeront immanquablement, à leurs yeux, 
les possibilités de réussite sociale, déjà jugées maigres. Chez ces jeunes, pour la plupart issus de milieu populaire, le capital corporel représente le plus grand espoir de réussite professionnelle (Boltanski, 1971). Ce d'autant plus que la voie de la légitimation scolaire, qui pourrait permettre un éloignement des tâches à dominante manuelle, leur est difficile d'accès (Esterle-Hédibel, 1995).

31 Si la recherche de profits symboliques par le biais de la prise de risque (en général) est omniprésente ${ }^{22}$, quel que soit le public, cette idée de gain prend une dimension matérielle chez les jeunes socialement instables (professionnalisation sportive, profit financier, aide pour trouver un emploi, etc.). Pour certains jeunes en situation d'échec ou de « chute » scolaire, la pratique sportive est même envisagée comme un substitut à la qualification scolaire («C'est pour çà que j'veux réussir dans l'basket, parce qu'à l'école laisse tomber !»). Ce désir de reconnaissance et de réussite par le sport justifie des efforts annexes, comme la pratique de la musculation ou l'inscription en club. On se trouve dans une logique de marché (recherche de profits, situations concurrentielles ...). Le sport occupe donc une place sérieuse, presque professionnelle avant l'heure pour certains de ces jeunes, opposée à l'image de " perte ", d'apparente gratuité des "sports à risque». Opposition bien connue entre les valeurs matérialistes, en vigueur notamment au sein des groupes sociaux ne jouissant pas d'un grand confort socioéconomique, et les valeurs post-matérialistes ou ludiques des groupes plus favorisés (Ball, 1972 : Inglehart et Abramson, 1994).

Ces «sportifs de rue " sont à la recherche, pour diverses raisons, d'une quelconque forme de réussite. L'usage fait du sport est logiquement tourné vers ce pôle, avec des modalités et des prises de risque qui lui sont propres. Il s'agit de stratégies identitaires, comme a pu les définir Rey (1993, p. 226) : «tentative de réaliser et de réussir une action individuelle dans un contexte de jeu très difficile et en prenant donc des risques. » Cela se traduit de diverses façons: tentatives de "shoot » en déséquilibre ou à trois points au basket, dribbles devant ses buts en foot, tentatives de gestes acrobatiques, spectaculaires, etc. Il s'agit de devenir, l'espace d'un instant, unique.

Influence des variables situationnelles

Avant tout social, interactionnel, le support contextuel des prises de risque se caractérise également par des relations particulières à certains lieux. Deux aspects ont été retenus : l'influence du groupe sur les comportements individuels et la mise en scène de soi, sciemment effectuée, qui régit une forme de " communication totale ».

Dans la majorité des cas une grande importance sera accordée à la possibilité d'être observé. Il est fréquent, notamment chez les skateurs ou les pratiquants du "roller ", de répéter les gestes techniques avant de se montrer en spectacle, une fois qu'ils sont bien maîtrisés. Ce qui permet de ne pas faire « mauvaise figure » en public. ("Tout seul on a pas la stress, on se sent pas regardé... On tombe c'est pareil») ( J'essaie un peu tout seul, puis quand j'sais l'faire moyennement j'essaie devant tout l'monde. »). On retrouve les notions de représentation, de région postérieure (où a lieu la répétition) et de région antérieure (où l'on se donne à voir) chères à Goff man (1973) et à son approche dramaturgique. La « rampe » de skate de Clermont-Ferrand, par exemple, est non seulement connue dans ses moindres recoins, mais également au niveau de sa fréquentation, des " heures de pointe ». La logique est semblable pour les pratiquants $\mathrm{du}$ «street-ball» («J'aime bien quand y'a du monde qui regarde, tout ça.../... Ca fait plaisir Si y'a personne, laisse tomber !») (« Le rêve, c'est de smasher: y'a d'quoi crâner, tout l'monde se lève, c'est trop bon !»). 

p. 196): «Il est clair que c'est pendant l'action que l'individu a l'occasion, dangereuse, de faire voir, à lui même et parfois à autrui, comment il se conduit quand les dés sont jetés. » Ces «caresses $»^{23}$ publiques ne sont cependant pas automatiquement recherchées, ou alors à travers d'autres interactions, comme celles existant au sein du groupe restreint, de la «tribu ». L'isolement ou le petit comité seront parfois jugés plus propices à la réalisation personnelle. Cette prise de distance par rapport à la possibilité d'être observé augmente en parallèle avec l'âge des interviewés.

Il est par ailleurs apparu que la logique des "hauts-lieux » était propre aux skateurs interrogés. Le "skate park ", situé en centre-ville, est à la fois une région antérieure et une région postérieure, selon le moment de la journée et l'affluence correspondante. Certains s'en éloignent, car "même si on y trouve des super sensations", ils n'apprécient pas l'aspect parqué de ce site. Dans la version «street » de la pratique, on s'amuse à se confronter à l'inadaptation de l'espace (escaliers, bancs, bordures, etc.). ( L'espace est aménagé, car créé par l'homme, mais on se sert de choses qui n'ont pas été faites pour...»). Des endroits explicitement interdits aux skateurs sont l'objet de " coups », en raison des possibilités de pratique ou de visibilité qu'ils recèlent ${ }^{24}$.

pratiquants du «street ball " interrogés n'ont pas, pour leur part, de terrain fédérateur, comme c'est le cas par exemple sur l'agglomération Lyonnaise (Camy/ Chantelat/Fodimbi, 1996). Ils jouent dans leur quartier, sur les terrains de proximité. $C^{\prime}$ est sans doute au rôle de reconnaissance locale de leur pratique que cette observation est due, car on discerne en effet chez eux un désir d'immédiateté des retombées sociales, à l'échelle du quartier. Leur mode de socialisation s'avère donc communautaire, primaire et local. A l'inverse, les «sujets à risque» sportifs expérimentent une socialisation beaucoup plus ouverte, basée sur des rapports de deux types: les premiers restreints au groupe de pairs (qui joue un rôle de miroir narcissique) et les seconds fragmentaires, mouvants, utilisant la figure de «l'étranger " (Simmel, 1908) pour lui communiquer l'image souhaitée, sans engagement particulier.

Principaux freins à la pratique de sports à risque corporel chez les jeunes sportifs socialement instables

Leur coût économique élevé25, fréquemment mis en avant, n'explique pas tout. D'autres facteurs, relatifs au quotidien des jeunes, à leur style de vie, bref à leur culture viennent entraver la pratique de ces activités. Tout d'abord, l'offre sportive est souvent des plus réduites pour les jeunes de "cité ", qui ne disposent pas pour la plupart de moyens de transport et doivent par conséquent composer avec les possibilités de pratique locale. («Dans l'quartier, y'a qu'un terrain de basket, y'a qu'ça! On a rien à faire, alors... »). Les jeunes "en galère », ceux qui sont le plus en difficulté, restent en marge des actions sociales leur étant destinées, et qui pourraient au moins ponctuellement leur faire découvrir des pratiques sportives originales ${ }^{26}$ («Quad» ou moto à quatre roues, saut à l'élastique ...).

De plus, l'intérêt porté pour ces pratiques à risque est parfois tout simplement relatif, en comparaison avec ce que peut apporter par exemple celle du basket. A ceci plusieurs raisons, Tout d'abord, le niveau d'excellence dans ces disciplines parait difficilement atteignable. Le calcul est en effet favorable aux sports traditionnels quant aux chances d'atteindre un niveau correct, point qui conditionne pour beaucoup l'engagement dans une pratique sportive (Duret, 1995). Le prix du matériel, des séances d'apprentissage, des transports éventuels, associés au peu de vécu technique dans ces activités 
alternatives contribuent donc à les rendre peu attirantes pour ce public particulier Le point de vue d'un éducateur du P.A.R.Q. 10 proposant parfois des activités sportives à risque aux jeunes accrédite ce constat. Les jeunes en difficulté acceptent, à ses yeux, moins bien les situations d'échec, inévitables dans les sports à risque (surtout en période de prise de contact avec l'activité), que les jeunes bien insérés, ce en raison de leurs parcours marqués du sceau de l'échec (notamment scolaire). Les pratiques de loisir doivent, au contraire, dans leur optique, contribuer à les mettre en situation potentiellement positive, valorisante.

Le sentiment de précarité de la santé, qui génère par ailleurs un élargissement de la perception du risque (cf. supra), fait de celle-ci un capital à préserver plutôt qu'à consumer. Il semblerait que l'habitus corporel décrit par Boltanski (1971), générateur selon Esterle-Hédibel (1995) de conduites à haut risque physique, soit difficilement applicable à ces jeunes. D'autant plus que leurs espoirs de réussite grâce au sport passent par une hygiène de vie irréprochable.

41 Enfin, l'attrait exercé par le milieu naturel est parfois des plus limités chez ces jeunes au mode de vie complètement associé à la ville, et dans l'univers culturel desquels la nature n'est pas des plus signifiantes.

Conclusion

42 Il est temps à présent de rappeler les principales avancées que ce travail a permises. La nécessaire concision de cette contribution aura en effet pu obscurcir le propos présenté. Il semble important d'insister sur quatre points.

43 Tout d'abord, il s'avère viable, comme nous en formulions la possibilité, de parler d'un champ de la prise de risque juvénile. La diversité de ses éléments constitutifs (corporels ou non, sportifs ou extra-sportifs) force l'élargissement de la définition classiquement associée d'une part aux « sports à risque » et d'autre part aux « conduites à risque » de la jeunesse. Ce décloisonnement, à partir d'une définition «ouverte» du risque, est la condition première de toute approche globale du phénomène étudié. La mise en lumière de stratégies et de déterminismes autour de l'objet d'étude confirme si besoin est la pertinence de cette première conclusion.

Deuxièmement, un enjeu est perceptible autour de la définition du risque, apparaissant par exemple dans le côté exagéré, déformé de certaines déclarations relatives à l'engagement dans des pratiques à risque. Que ce décalage soit sciemment construit (s'inscrivant dans une logique emphatique) ou qu'il soit le fruit de représentations sociales (rapprochant leurs auteurs de la «schizophrénie sportive » abordée), il justifie le choix de ne pas restreindre a priori la portée sémantique du terme en question. Il existe des conflits, des positionnements, bref tout un jeu autour de la notion de risque. $C^{\prime}$ est ce qui rend nécessaire la prise en compte des multiples visions formulées par le public plutôt que d'une définition universelle et objective du concept.

La première hypothèse de cette recherche prônait une nouvelle approche, que l'on peut qualifier de conjonctive, du champ de la prise de risque juvénile. Il ne suffit plus de considérer l'actuel engagement des jeunes dans les multiples comportements alternatifs mis en exergue suivant une logique unique. C'est ce qui justifie la présence de références à des auteurs que tout oppose a priori (le champ de référence, les paradigmes explicatifs, l'époque...). Cette perspective anthropologique, la moins restrictive possible, est ainsi censée couvrir les différents usages faits de la prise de risque par les jeunes. 
Enfin, l'approche de terrain mise en place, dictée par la prise en compte de l'« expérience », a révélé la pluralité des constructions sociales conduisant à l'adoption de comportements risqués, attestant de la complémentarité des options théoriques abordées. L'héritage des "pionniers » de la sociologie s'est avéré aussi utile dans cette optique que les avancées plus récentes, interactionnistes ou postmodernistes par exemple. Il s'avère désormais nécessaire d'appréhender le champ du risque de manière moins simpliste, en complexifiant la question, à moins de circonscrire à outrance la définition des comportements dits risqués. Si l'on peut toujours parler de correspondance entre niveau d'intégration sociale et forme de confrontation au risque, il faut souligner les spécificités de celles-ci. Les antécédents relatifs aux conduites de chaque groupe sont parfois mêlés, rarement tranchés et binaires. Certains points communs aux deux groupes émergent de ce travail: le risque comme prétexte à la recherche de sensations, le rôle activateur du groupe de pairs, ou encore la recherche de «mise en scène » des prises de risque sportives. Ce qui n'exclut pas les divergences. Les « sujets à risque » sportifs, sans grande surprise, sont les plus représentés au niveau des variables relevant $d u$ risque dynamique (stratégies distinctives, émotions collectives au sein du groupe restreint, recherche d'une meilleure connaissance de soi ...). Cela dit, des « déterminismes inversés " guident aussi leurs actes. Ils traduisent une recherche de rupture avec la routine quotidienne, mortifère, et la nécessité de se constituer une marge de liberté. Une forme d'instabilité psychologique, plutôt que sociale, est parfois perceptible chez les interviewés de ce groupe. Les prises de risque des jeunes socialement instables sont tout aussi bien imputables à la recherche de bénéfices (désir de reconnaissance sociale, rêve de carrière ...) qu'à un palliatif face à la fragilisation sociale dont ils sont victimes. Ils cherchent alors à travers elle à montrer qu'ils ne sont pas toujours exclus du monde des performants, des vainqueurs, ce qui passe par des modalités particulières de prises de risque sportives. La subtilité des différences réellement constatées justifie bien l'affinement de la question traitée. Et c'est bien grâce à l'approche microsociologique, conformément à la seconde hypothèse avancée, que l'on a pu mettre à jour ces « bricolages » en oeuvre autour du risque.

On peut pour conclure affirmer que le risque est un élément fort, qui ne peut laisser indifférent, a fortiori lorsqu'il touche l'intégrité physique des personnes. Ses capacités signifiantes en font un support privilégié, dans le domaine sportif notamment, de la construction identitaire. Le caractère digital de ses «délibérations » et les nombreux échanges qui gravitent autour de ses divers visages en font un support potentiellement structurant et socialisant. Une alternative à l'attitude prophylactique générée par les conduites à risque émerge de cette réflexion. Tournée vers l'éradication de ces comportements, celle ci peut être agrémentée d'une tentative de transfert des prises de risque déviantes en prises de risque fécondes, dont l'existence à l'intérieur du même champ a été démontrée : "Contre les suicides et contre toutes les conduites ordaliques de notre société (délinquance, toxicomanie, vitesse, etc.), il faut replacer le risque dynamique contre le risque ordalique : c'est-à-dire le risque que quelqu'un prend afin d'augmenter le sens du réel pour lui-même et la société, plutôt que le risque ordalique où l'on attend que les autres tranchent à notre place. » (Dolivet, 1994, p. 166). 


\section{BIBLIOGRAPHIE}

Assailly J-P (1992) Les jeunes et le risque : une approche psychologique de l'accident. Paris, Vigot.

Assailly J-P (1990) Le risque accidentel à l'adolescence et sa prévention. Vol. Il Le concept de risque et la genèse $d u$ risque accidentel chez les jeunes. Rapport I.N.R.E.T.S. $\mathrm{N}^{\circ} 131$

Aubert N. et De Gaulejac V (1991) Le coût de l'excellence, Paris, Éditions du Seuil.

Ball D.W. (1972) What the action is: a crosscultural approach : Journal for the theory of social behaviour, 2, Vol. 2.

Bardin L. (1980) L'analyse de contenu. Paris, PU.F.

Baudry P (1991) Le corps extrême. Approche sociologique des conduites à risque. Paris, L'Harmattan.

Bazin H. (1995) La culture hip hop. Paris, Desclée de BrouwIer.

Berne E. (1967) Des jeux et des hommes. Psychologie des comportements humains. Paris, Stock.

Boltanski L. (1971) Les usages sociaux du corps : Annales Economie Société Civilisation, 1, Paris, 206-233.

Bourdeau P. (1995) L'escalade, entre sport et tourisme. Synthèse du rapport réalisé pour l'Agence Française de l'Ingénierie Touristique. A.F.I.T.

Camy J., Chantelat P., Fodimbi M. (1996) Sports de la cité. Anthropologie de la jeunesse sportive. Paris, L'Harmattan.

Choquet M. (1991) Le risque à l'adolescence, à partir des enquêtes épidémiologiques, Cahiers de la sécurité intérieure, 5, 45-58.

Collard L. (1997) Approche sociologique des sports à risque : Revue S.T.A.P.S., 44, décembre 1997, 83-95.

Corcuff P. (1995) Les nouvelles sociologies. Constructions de la réalité sociale. Paris, Nathan.

Corneloup J. (Il 993) Escalades et sociétés. Contribution à l'analyse du système, du communicationnel et du social. Thèse de doctorat, Paris sud-Orsay.

Corneloup J. (1997) Risque, opinion publique et pratiques d'escalade, Société, n55, Paris, Dunod, 23-40.

Csikszentmihalyi M. (1977) Beyond boredom and anxiety. San Francisco, Jossey-Bass publishers.

Delignières D. (1993) Risque préférentiel, risque perçu et prise de risque : Cognition et Performance, Paris, I.N.S.E.P, 89-102.

Dolivet P (1995) Risques ordaliques; contre risques dynamiques, : Anstett M. et Sachs B. (dir.) Sporfs, jeunes et logiques d'insertion, Paris, La documentation Française, 163-183.

Dubet F. (1994) Sociologie de l'expérience. Paris, Editions du Seuil.

Duret P (1996) Anthropologie de la fraternité dans les cités. Paris, PUF

Ehrenberg A (1991) Le culte de la performance. Paris, Calmann Levy.

Elias N., Dunning E. (1994) Sport et civilisation. La violence maîtrisée. Paris, Fayard.

Esterle-Hédibel M. (1995) Le rite et le risque, la culture du risque dans les bandes de jeunes de milieu populaire à travers la conduite routière. Thèse de doctorat, Paris V (Université R. Descartes). 
Fournier A., Guitton C., Kervern, G.Y. et al.(1996) Le risque psychologique majeur. Introduction à la psychosociologie cindynique. Paris, Editions ESKA.

Galland 0. (1991) Sociologie de la jeunesse. L'entrée dans la vie. Paris, Armand Colin.

Goffman F. (1973) La mise en scène de la vie quotidienne. Paris, Editions de Minuit.

Goffman F, (1974) Les rites d'interaction. Paris, Editions de Minuit.

Griffet J (1991) La sensibilité aux limites : Griffet J. (dir.) Sociétés, 34, Paris, Dunod, 359-365.

Hirschi T. (1969) Causes of delinquency. Berkeley, University of California press,

Inglehart R., Abramson P.R. (1994) Economic security and value change : American political science review, Vol. 88, $\mathrm{N}^{\circ}$ 2, University of Michigan (USA), 336-354.

Jackson D.N, Hourany, B, Vidmar, N.J. (1972) A four-dimensional interpretation of risk taking :

Journal of Personality, 40, 483-501.

Jessor R. (1987) Risky driving and adolescent problem behaviour: an extension of Problem Behaviour Theory : Alcohol, Drugs and Driving, 3, 1 -11.

Jessor R.; Donovan, J.E.; Costa, F. (1989) Problem drinking and risky driving among youth : a psychosocial approach to a lifestyle pattern : Actes l.N.R.E.T.S.,18, 137-152.

Klausner S.Z. (1968) Why man take chance. Studies in stress-seeking, Anchor, Garden city doubleday. Le Breton D. (Il 991) Passions du risque. Paris, Métailié.

Le Breton D. (1995) La sociologie du risque. Paris, PUF

Lopes L.L. (1983) Some thoughts on the psychological concept of risk: Journal of Experimental Psychology, 9,137-144.

Loret A. (1997) Le sport en mouvement, Cultures en mouvement, 2, avril-mai 1997, 34-37.

Loret A. (1995) Génération glisse. Dans l'eau, l'air la neige... La révolution du sport des années fun. Paris, Editions Autrement (collection Mutations).

Lyng S. (1990) A social psychological analysis of voluntary risk taking : American Journal of Sociology, vol.95, No 4.

Maffesoli M. (1993) La contemplation du monde : figures du style communautaire. Paris, Grasset.

Maffesoli M. (1991) Du nomadisme, Griffet J. (dir.) Sociétés, 34, Paris, Dunod.

Marange V (1995) Les jeunes. Paris, Le Monde Editions.

Marc E., Picard D. (1989) L'interaction sociale. Paris, .PU.F

Michon B., Ohl F (1993) Marketing et sociologie : analyse de la consommation des produits Sportifs, in : Loret A, (clin) Sport et management. De l'éthique à la pratique, Paris, Dunod, 208-233.

Mitchell R.G. Jr (1983) Mountain experience. The psychology and sociology of aventure. Chicago, The University of Chicago press.

Mucchielli A. (1991) Les méthodes qualitatives. Paris, .PU.F.

Ohl F. (1993) Consommations sportives et interactions sociales : les articles de sport, Actes du Colloque des 14 et 15 octobre 1993 à Bordeaux : Sport, relations sociales et action collective, Talence, M.S.H.A, 675-684.

Padis M.O. (1996) La génération miroir : Esprit, 225, octobre 1996, 5-7.

Pociello C. (1992) L'aventure : pratiques et représentations : Universalia 1992, 346-352. 
Pociello C. (1995) Les cultures sportives. Paris, PUF

Rey J.-P. (1993) Conduites de jeu et stratégies identitaires : une tentative explicative des comportements risqués dans le jeu sportif collectif, Actes du Colloque de Bordeaux des 14 et 15/10/ 1993 : Sport, relations sociales et action collective, Talence, M.S.H.A., 223-229.

Sachs B. (1990) En guise de synthèse : Vertiges, Sports à risque et Toxicomanie, Marseille, A. M. P. P, 133-137.

Soulé B. (1997) Jeunes et activités sportives à risque: vers une approche sociologique contextualisée. Mémoire de D.E.A. (dir. J. Corneloup). U.F.R. S.T.A.P.S. Clermont-Ferrand.

Tap P. (1996) Marquer sa différence : Sciences Humaines hors-série, N15, décembre 1996-Janvier 1997, 9-10

Therme P. (1995) L'échec scolaire, l'exclusion et la pratique sportive. Paris, PUF

Zuckerman M. (1979) Sensation seeking. Beyond the optimal level of arousal. Hillsdale (U.S.A.), Laurence Erlbaum.

\section{NOTES}

1. Il s'agit, entre autres, de l'escalade, du surf, de la « glisse » hors-piste, du VTT., du skate, du « roller »... Depuis vingt ans, c'est un large mouvement souterrain qui redéploie tous les référentiels culturels du sport sous la bannière de la marginalité et de l'irrévérence (...) il semble bien que le "délire" ait pris le pas sur la raison. La légitimité va au vertige. Stupéfiante, dès lors, devient la recherche forcenée du comportement incertain, du degré improbable, du niveau problématique. » Alain Loret : Le sport en mouvement (voir bibliographie).

2. La culture sportive de la jeunesse lui fait désormais la part belle. Christian Pociello parle de «fait porteur d'avenir » à propos de cette mise sous forme aventureuse des pratiques, qui s'affirme selon lui comme un élément primordial à prendre en compte dans l'étude sociologique du champ sportif (Les cultures sportives : voir bibliographie). 3. David Le Breton « Depuis une vingtaine d'années, l'émergence de conduites nouvelles liées aux prises de risque, à l'exposition de soi à l'adversité, modifie sensiblement la sociologie de la jeunesse. La mortalité et la morbidité qui touchent cette classe d'âge sont préoccupantes. » La sociologie du risque (voir bibliographie).

4. La Fédération Nationale de la Mutualité Française a établi en 1988 un classement des sports les plus traumatisants pour 100 pratiquants : arrivent en tête et dans l'ordre la boxe, le rugby, le hockey sur glace, le handball, le bobsleigh, la lutte, le football, le basket-ball, le hockey sur gazon et le judo (source : Fédération Nationale de la Mutualité Française, 1988, Paris).

5. $80 \%$ des lycéens estiment probable de se retrouver un jour au chômage d'après un sondage Phosphore/CSA réalisé en septembre 1993 (Jean- Michel Dumay, Le Monde du 27/05/1994).

6. Nous inspirant en cela de la définition de l'intégration sociale donnée par Sciences Humaines (hors série « Le lien social en crise »), mai-juin 1996,

7. «Conduites volontaires et délibérées, accomplies lucidement conduites répétitives, hors d'un cadre professionnel (sportif ou professionnel par exemple) ' conduites entraînant un danger de mort ou de lésion grave pour le sujet, et dont l'enjeu allégué (plaisir, profit, passion ou autre) parait à l'observateur disproportionné au risque 
encouru. Enfin, conduites qui laissent au hasard une large part, voire toute la place. » in Anne Fournier, Catherine Guitton, Georges-Yves Kervern et al. Le risque psychologique majeur (voir bibliographie).

8. D'après l'analyse du risque routier réalisée par Jean-Pascal Assailly (voir bibliographie).

9. L'I.N.S.E.R.M. a annoncé en 1994 un taux de mortalité dû au suicide chez les 15-24 ans de $15 \%$. contre $10 \%$ en 1979 . Ce, malgré le tabou qui entoure encore les décès par suicide (seul un sur cinq serait déclaré comme tel). « La santé en France en 1996 », 1996, Documentation Française, Paris.

10. D'après les éducateurs du 1 de Clermont-Ferrand (Prévention, Action, Rues et Quartiers, service spécialisé de l'A.D.S.E.A. (Association Départementale de Sauvegarde de l'Enfance et de l'Adolescence). Les éducateurs en question sont MM, Brunhe et Bouet.

11. Enquête de l'I.N.S.E.R.M. (1993), en collaboration avec le ministère de 'Education Nationale, sur la santé des collégiens et lycéens.

12. Données sociales 1996 - I.N.S.E.E.

13. C'est ce que déplore Philippe Bourdeau « ... rares sont les données fiables et homogènes permettant par exemple de comparer la "dangerosité" des différents types de sport ( ... ) L'accès à un tel matériau montrerait d'ailleurs peut-être que les sports de montagne, organisés autour de la symbolique et de la réalité du risque sont moins dangereux - toutes proportions gardées - que bien d'autres activités sportives jugées banales. » in « Le statut ambigu du risque dans les sports de montagne » (voir bibliographie).

14. La Mutuelle Nationale des Sports a enregistré 101 décès parmi ses membres en 1995 , contre 79 en 1994, 64 en 1990 et 45 en 1986, alors que son nombre d'adhérents a chuté d'un million ! 1. Giroire, directrice adjointe: « Les pratiques les plus inquiétantes sont l'alpinisme (...) et le vélo, dont la version tout-terrain est beaucoup plus dangereuse qu'on ne le pense. » Même écho à l'union Mutuelle des Sportifs, où l'on se déclare tout aussi inquiet devant les sinistres liés à ces pratiques. (Le Monde du 20 et 21/10/1996). Paul Irlinger («La France sportive: entre pratiques et représentations » Universalia 1989, pp. 380-385) confirme la tendance : « Le traditionnel dépassement de soi dans l'effort n'est plus qu'une direction de développement parmi d'autres, concurrencée sur son propre terrain par de nouvelles ascèses sportives (triathlon, courses longues sur routes...) et des pratiques à haut risque, aventures sportives dont le Paris-Dakar constitue la plus spectaculaire. »

15. Remarque formulée par Marie Choquet (voir bibliographie).

16. D'après les enquêtes épidémiologiques rapportées par Jean-Pascal Assailly (1992) (voir bibliographie).

17. D'après une enquête pour le Comité Français d'Education pour la Santé réalisée par I.P.S.O.S. en 1996 auprès de 1006 adolescents (Libération, 30/05/1995).

18. Selon Jean-Pascal Assailly (voir bibliographie).

19. D'après les mêmes éducateurs du PA.R.Q. de Clermont-Ferrand.

20. Enquête de l'association D.E.Fl. avec l'institut Louis Harris, auprès des jeunes de moins de 25 ans en situation précaire.

21. Une enquête de l'I.N.S.E.E. réalisée en 1991 et 1992 dévoile que 93\% des hommes et $85 \%$ des femmes de 18 à 24 ans se déclarent en bonne ou très bonne santé.

22. La volonté, chez les « risqueurs » sportifs, de projeter sur leur entourage une expression amplifiée du caractère risqué de leur pratique n'est pas forcément 
volontaire, dans le sens où certains d'entre eux paraissent convaincus du caractère dangereux de leur activité (qu'une observation objective, in situ, rendra pour partie d'entre eux exagéré). Les figures emblématiques portées aux nues par les médias, les fantasmes de l'extrême qui y sont associés générant cette schizophrénie sportive, par un mécanisme de mimétisme naiff et d'identification excessive. Cette logique du «faux semblant » pourrait expliquer certaines surévaluations flagrantes du risque corporel couru, relativisant du même coup son utilité stratégique.

23. Terme utilisé par Eric Berne (voir bibliographie) pour qualifier les compliments, les comportements attendus, espérés suite à l'action.

24. C'est la même logique qui pousse les « taggeurs » à rechercher des lieux à fort impact visuel, parfois au risque de leur intégrité corporelle Hugues Bazin : La culture hip hop (voir bibliographie).

25. La catégorisation des sports selon leur coût annuel établie par Bernard Michon et Fabien Ohl fait apparaître les activités à risque comme étant les plus onéreuses (voir bibliographie)

26. Celles-ci sont pourtant réservées en priorité aux jeunes présentant leur profil, mais sont « trustées " par des jeunes sans problème majeur qui font eux la démarche de s'inscrire dans les projets établis.

\section{RÉSUMÉS}

Ce travail constitue une tentative de compréhension de l'engouement perceptible, chez certains jeunes, pour des pratiques sportives dites risquées. L'étude de cette modalité particulière de prise de risque corporelle, simple élément du plus vaste champ des conduites à risque juvéniles, a permis d'aboutir au constat de la diversité des constructions sociales de la prise de risque. Ce propos se polarisera sur la stratification sociale du rapport au risque et des prises de risque corporelles de la jeunesse, et sur l'importante capacité communicationnelle du risque sportif, génératrice d'une forme particulière de lien social.

This research workforms an attempt to understand young people infatuation for physically risked sports. The case of sports risk taking, simple component of the larger youth riskbehaviour field, enabled us to lead up in the result of social constructions variety of risk taking. We'll focus here on social stratification of young people physically risked behaviours, and sports risk's high communication virtues, creative of a specific form of social link.

\section{INDEX}

Keywords : context, risk taking, social integration, sport, sports, youth

Mots-clés : contexte, intégration sociale, jeunesse, prise de risque 


\section{AUTEURS}

\section{BASTIEN SOULÉ}

Faculté des Sciences du Sport et de l'Education Physique. Université de Paris Sud-Orsay. C. R. C. S.

JEAN CORNELOUP

Faculté des Sciences du Sport et de l'Education Physique. Université de Clermont II. Laboratoire

d'anthropologie des pratiques corporelles 